\title{
A Unified and Elementary Proof of Serial and Nonserial, Univariate and Multivariate, Chernoff-Savage Results
}

\author{
D. Paindaveine ${ }^{1}$ \\ Département de Mathématique, I.S.R.O., and E.C.A.R.E.S., Université Libre de \\ Bruxelles, Campus de la Plaine CP 210, B-1050 Bruxelles, Belgium
}

\begin{abstract}
We provide a simple proof that the Chernoff-Savage [1] result, establishing the uniform dominance of normal-score rank procedures over their Gaussian competitors, also holds in a broad class of problems involving serial and/or multivariate observations. The non-admissibility of the corresponding everyday practice Gaussian procedures (multivariate least-squares estimators, multivariate $t$-tests and $F$-tests, correlogram-based methods, multivariate portmanteau and Durbin-Watson tests, etc.) follows. The proof, which generalizes to the multivariate - possibly serial - setup the idea developed in Gastwirth and Wolff [2] in the context of univariate location problems, allows for avoiding technical convexity and variational arguments.
\end{abstract}

Key words: Pitman-inadmissibility, rank-based inference, Chernoff-Savage results, multivariate signs and ranks.

$1991 M S C: 62 \mathrm{G} 20$.

\section{Introduction.}

\subsection{Uniform efficiency of classical rank-based procedures.}

Since their introduction in the beginning of the 20th century, rank-based methods have met much success, due to their many advantages over stan-

\footnotetext{
Email address: dpaindav@ulb.ac.be (D. Paindaveine).

URL: http://homepages.ulb.ac.be/ dpaindav (D. Paindaveine).

1 Research supported by a P.A.I. contract of the Belgian federal Government, and an A.R.C. contract of the Communauté française de Belgique.
} 
dard Gaussian methods. Unlike Gaussian procedures, the rank-based ones are indeed purely nonparametric, and therefore allow for avoiding the somewhat artificial and questionable specification of the distribution of the noise associated with the model under study (aiming at easier analytic derivations, rather than addressing any real modelling issue). Rank procedures also are distribution-free and are generally valid under broader conditions than the parametric Gaussian ones (in the one-sample location case, for instance, rank tests are valid without any moment assumption, whereas standard $t$-tests require finite second-order moments). Last but not least, rank-based methods are intrinsically more robust than their parametric competitors.

While these advantages of rank-based methods are usually well recognized in the statistical community, it is generally thought that, intuitively, too much information is thrown away when using the observations through their ranks (and/or signs) only, and that, consequently, the price to pay for the nice properties above is a severe efficiency loss. In that respect, two striking results, which show that intuition, in this case, is totally misleading, had quite an impact on the success and subsequent development of rank-based inference.

The celebrated Hodges-Lehmann [3] ".864 result" states that the lower bound, in the two-sample location model (but this extends to most location problems, such as one-sample, $c$-sample, ANOVA, regression problems, etc.), of the Pitman asymptotic relative efficiency (ARE) of Wilcoxon (i.e., linear scores) rank tests with respect to their normal-theory competitors (namely, standard two-sample $t$-tests) is .864. In other words, Wilcoxon tests, asymptotically never - that is, whatever the distribution of the underlying noise - need more than $14.6 \%$ observations more than $t$-tests to achieve the same power (see, e.g., Pratt and Gibbons [4] for a more formal definition of ARE).

No less celebrated is the result by Chernoff and Savage [1] proving that the lower bound, in the same type of models, of the ARE of normal-score rank tests, still with respect to the corresponding standard Gaussian tests, is 1, and that this lower bound is reached at Gaussian distributions only. This means that not only do (ad hoc versions of the) rank-based procedures perform equally well as the Gaussian procedures in the normal case (where the latter are known to be optimal), but they are strictly more efficient than Gaussian procedures as soon as the underlying distribution is not normal. The inadmissibility - in the Pitman sense of the standard Gaussian procedures follows. Gastwirth and Wolff [2] gave a simple proof of this result, mainly based on Jensen's inequality, which allows for avoiding the technicalities in Chernoff and Savage's original paper.

Moreover, the above results deal with "worst cases"; both for the Wilcoxon and the normal-score rank tests, it is possible to show that there is no "best case", that is, it is possible to find sequences of underlying distributions along 
which the corresponding ARE's (still with respect to standard Gaussian tests) go to infinity. These considerations not only dispel the fears of poor efficiency of rank-based methods, but also show that efficiency actually is another advantage of rank-based methods over the classical Gaussian ones.

Finally, it should be stressed that the rank-based procedures that beat uniformly the standard ones are not just theoretical refinements of the latter, with marginal benefits (such as, e.g., shrinkage estimators) : they are practical, readily implementable solutions to actual problems, bringing sizeable improvements over traditional methods.

\subsection{Recent advances in rank-based inference and generalized Chernoff-Savage results.}

The original Chernoff-Savage [1] result is restricted to univariate location models. The reason for this is not that the uniform dominance of normal-score rank procedures over parametric Gaussian ones is some kind of miracle that is specific to univariate location models, but rather that rank-based methods for a long time have been essentially limited to statistical models involving univariate independent observations. Except for a few exceptions (such as testing against bivariate dependence, tests based on runs, tests for scale, or goodness-of-fit methods that do not address any specific alternative), classical monographs (Hájek and Šidák [5] and Hájek, Šidák, and Sen [6]; Lehmann [7]; Randles and Wolfe [8]; Pratt and Gibbons [4]; Hettmansperger [9]; Puri and Sen [10] - to quote only a few) mainly deal with single-response linear models with independent errors: one- and two-sample location, analysis of variance, regression, etc.

In particular, despite the fact that some of the earliest and most classical rank tests - such as runs tests, turning point tests, etc. - are addressing problems of serial dependence and thus are of a genuine serial nature, no systematic and coherent theory of serial rank-based statistics was constructed until the mideighties, where such statistics were considered in a series of papers (Hallin et al. [11], Hallin and Puri [12-14]; see Hallin and Puri [15] for a review of rankbased testing in a (univariate) ARMA context). Hallin [16] extended Chernoff and Savage's result to this serial context by showing that the normal-score version of his serial rank tests also uniformly dominates the corresponding Gaussian competitors (standard portmanteau tests, Durbin-Watson tests, etc.).

In the same spirit, although it has attracted much attention in the late fifties and the sixties, leading to a fairly complete theory of hypothesis testing based on componentwise ranks (a unified account of this line of research is given in the monograph by Puri and Sen [17]), the extension of rank-based methods to 
problems involving multivariate observations was even slower than their extension to the serial context. The componentwise approach indeed suffers the major weakness of being based on the choice of a particular coordinate system, and it took about twenty years to see the emergence of a systematic development of coordinate-free, affine-invariant competitors to these componentwise sign and rank methods.

This development, initiated in the late eighties, essentially expanded along two distinct lines of research. The first one, based on Oja signs and ranks, is due to Oja, Hettmansperger, and their collaborators (Möttönen et al. [18-20]; Hettmansperger et al. [21,22]; see Oja [23] for a review). The second one is associated with ranks of Mahalanobis distances and Randles' concept of interdirections, and was developed by Randles and his coauthors (Randles [24,25]; Peters and Randles [26]; Jan and Randles [27]; Randles and Um [28]). Hallin and Paindaveine [29-31] proposed fully coordinate-free testing proceduresbased on signs and ranks of this second type - for a broad class of problems in the multivariate general linear model with (elliptically symmetric) vector ARMA errors. As proved in Hallin and Paindaveine [29,30], the ARE's of the normal-score version of their procedures, still with respect to the parametric Gaussian ones (Hotelling's $T^{2}$ tests, multivariate portmanteau tests, etc.), are uniformly larger than 1, which shows that Chernoff-Savage's result extends to the multivariate (possibly serial) set-up as well.

It is remarkable that Chernoff-Savage results still hold in those multivariate location and multivariate serial problems, showing that the corresponding everyday practice Gaussian procedures (multivariate least-squares, $t$-tests, $F$-tests, correlogram-based methods, multivariate Portmanteau and DurbinWatson tests, etc.) are not admissible in the Pitman sense. Also, as in the univariate case, the rank-based procedures defeating uniformly the standard ones are not just theoretical refinements of the parametric Gaussian procedures, but are easily implementable, theoretically and practically appealing, solutions to actual problems.

\subsection{Outline of the paper.}

Our main goal in this paper is to provide an elementary and unified proof that the Chernoff-Savage phenomenon indeed holds in a broad class of problems involving serial and/or multivariate observations. The proof, which generalizes to the multivariate - possibly serial - set-up the idea developed in Gastwirth and Wolff [2], allows for avoiding the technical convexity and variational arguments used in Hallin and Paindaveine [29,30]. For the sake of simplicity, we mainly focus on two generic multivariate problems, in which we prove Chernoff-Savage results; for reasons that are given in the final section, these 
particular Chernoff-Savage results automatically extend to a much broader set-up.

The outline of the paper is as follows. In Section 2, we introduce the two generic multivariate problems considered, namely the one-sample location problem and the problem of testing for multivariate randomness against vector ARMA dependence, both in their elliptically symmetric versions. In Section 3, we state the corresponding Chernoff-Savage results and give our elementary proof. We make some final comments in Section 4, which more explicitly describes the non-admissibility consequences - which go far beyond the two particular cases considered in Section 2-of the Chernoff-Savage results of which we give an elementary proof.

\section{Two generic problems.}

We will consider Chernoff-Savage results both in a multivariate location and in a multivariate serial set-up. In each case, we will restrict to an elliptically symmetric problem. Recall that the distribution of a centered random $k$-vector $\mathbf{X}$ is said to be elliptically symmetric with "parameters" $\Sigma$ and $f$ if and only if its pdf is given by

$$
\underline{f}_{\boldsymbol{\Sigma} ; f}(\mathbf{x}):=c_{k, f}(\operatorname{det} \boldsymbol{\Sigma})^{-1 / 2} f\left(\left(\mathbf{x}^{T} \boldsymbol{\Sigma}^{-1} \mathbf{x}\right)^{1 / 2}\right), \quad \mathbf{x} \in \mathbb{R}^{k},
$$

for some symmetric positive definite real $k \times k$ matrix $\boldsymbol{\Sigma}=\left(\Sigma_{i j}\right)$ with $\Sigma_{11}=1$, and some function $f: \mathbb{R}_{0}^{+} \longrightarrow \mathbb{R}^{+}$such that $f>0$ a.e. and $\mu_{k-1 ; f}:=$ $\int_{0}^{\infty} r^{k-1} f(r) d r<\infty\left(c_{k, f}\right.$ is a normalization factor depending on the dimension $k$ and $f)$. We will denote this distribution by $\mathcal{E}_{k}(\boldsymbol{\Sigma}, f)$.

The shape parameter $\boldsymbol{\Sigma}$ determines the orientation and shape of the associated equidensity contours. Since all testing problems and tests considered in the sequel are invariant under affine transformations, the ARE's under study will not depend on the value of $\Sigma$, and we can restrict - without loss of generality - to the class of spherical distributions, for which $\Sigma$ coincides with the $k$-dimensional identity matrix $\mathbf{I}_{k}$.

Under $\mathcal{E}_{k}\left(\mathbf{I}_{k}, f\right)$, the radial density $f$ determines the distribution of $\|\mathbf{X}\|$. More precisely, the probability density function of $\|\mathbf{X}\|$ is $\tilde{f}_{k}(r):=\left(\mu_{k-1 ; f}\right)^{-1} r^{k-1}$ $f(r) I_{[r>0]}\left(I_{A}\right.$ stands for the indicator function of the set $\left.A\right)$; denote by $\tilde{F}_{k}$ the corresponding distribution function.

To guarantee that (1) is a density, we need to assume that $\mu_{k-1 ; f}<\infty$. When discussing inadmissibility issues of the various parametric Gaussian procedures (which require the underlying distribution to have a finite vari- 
ance), we will restrict to radial densities satisfying the stronger condition $\mu_{k+1 ; f}:=\int_{0}^{\infty} r^{k+1} f(r) d r<\infty$, under which the distribution $\mathcal{E}_{k}\left(\mathbf{I}_{k}, f\right)$ has finite second-order moments. One can associate with each radial density $f$ the density type of $f$ defined as the class $\left\{f_{a}, a>0\right\}$, where $f_{a}(r):=f(a r)$, for all $r>0$. By affine-invariance, one could restrict to couples of parameters of the form $(\Sigma, f)=\left(\mathbf{I}_{k}, f_{a_{0}}\right)$ for which the variance of the associated elliptical distributions is equal to $\mathbf{I}_{k}$. However, it will be convenient in the sequel to consider all possible radial densities, so that we will only fix $\boldsymbol{\Sigma}=\mathbf{I}_{k}$ but let $f$ range over its density-type. Some extremely mild smoothness conditions on $f$ - that we throughout assume to be fulfilled - are required to derive ARE's. See Hallin and Paindaveine $[29,30]$ for details.

The radial density $f$ is said to be Gaussian if and only if $f=\phi_{a}$ for some $a>0$, where $\phi(r):=\exp \left(-r^{2} / 2\right)$. Under $\mathcal{E}_{k}\left(\mathbf{I}_{k}, \phi\right)$, the pdf of $\|\mathbf{X}\|$ is $\tilde{\phi}_{k}(r):=$ $\left(2^{(k-2) / 2} \Gamma(k / 2)\right)^{-1} r^{k-1} \phi(r) I_{[r>0]}$ (where $\Gamma($.$) stands for the Euler gamma func-$ tion), and we denote by $\tilde{\Phi}_{k}$ the associated cdf. Under $\mathcal{E}_{k}\left(\mathbf{I}_{k}, \phi\right)$, the distribution of $\|\mathbf{X}\|^{2} \stackrel{\mathcal{D}}{=}\left(\tilde{\Phi}_{k}^{-1}(U)\right)^{2}$ (throughout, $U$ stands for a random variable that is uniformly distributed over $(0,1))$ is $\chi_{k}^{2}$, so $\tilde{\Phi}_{k}(r)=\Psi_{k}\left(r^{2}\right)$ for all $r>0$, where $\Psi_{k}$ denotes the distribution function of the $\chi_{k}^{2}$ distribution.

The multivariate location problem we consider is the multivariate elliptically symmetric one-sample location problem, for which, on the basis of the sample $\mathbf{X}_{i}=\boldsymbol{\theta}+\boldsymbol{\varepsilon}_{i}, i=1, \ldots, n$, where the $\boldsymbol{\varepsilon}_{i}$ 's are i.i.d. elliptically symmetric centered $k$-vectors, one wants to test $\boldsymbol{\theta}=\boldsymbol{\theta}_{0}$ against $\boldsymbol{\theta} \neq \boldsymbol{\theta}_{0}$, for some fixed $k$-vector $\boldsymbol{\theta}_{0}$. The classical parametric Gaussian test for this problem is Hotelling [32]'s $T^{2}$ test. Hallin and Paindaveine [29] developed a class of optimal signed-rank competitors of Hotelling's test. The ARE of the normal-score version of their tests, with respect to Hotelling's test, under radial density $f$, is given by

$$
\operatorname{ARE}_{k, f}^{(\mathrm{loc})}=\frac{1}{k^{3}} D_{k}(f)\left[C_{k}(\phi, f)\right]^{2}
$$

where, denoting by $\varphi_{f}:=-f^{\prime} / f$ the optimal location score function associated with the radial density $f$, we let

$$
C_{k}(\phi, f):=\mathrm{E}\left[\tilde{\Phi}_{k}^{-1}(U) \varphi_{f}\left(\tilde{F}_{k}^{-1}(U)\right)\right] \text { and } D_{k}(f):=\mathrm{E}\left[\left(\tilde{F}_{k}^{-1}(U)\right)^{2}\right]
$$

see Hallin and Paindaveine [29].

The benchmark multivariate serial problem we consider is that of testing for multivariate (elliptical) randomness against (elliptical) VARMA dependence. More precisely, we want to test the null hypothesis that the $k$-variate sample $\mathbf{X}_{1}, \mathbf{X}_{2}, \ldots, \mathbf{X}_{n}$ is the realization of an elliptically symmetric i.i.d. centered process; under the alternative, the sample is generated by some non-trivial 
VARMA model of the form

$$
\mathbf{X}_{t}-\sum_{i=1}^{p} \mathbf{A}_{i} \mathbf{X}_{t-i}=\varepsilon_{t}+\sum_{i=1}^{q} \mathbf{B}_{i} \varepsilon_{t-i}
$$

where $\mathbf{A}_{1}, \ldots, \mathbf{A}_{p}, \mathbf{B}_{1}, \ldots, \mathbf{B}_{q}$ are $k \times k$ real matrices and where the $\boldsymbol{\varepsilon}_{t}$ 's are i.i.d. elliptically symmetric centered $k$-vectors. Hallin and Paindaveine [30] proposed a class of multivariate signed-rank procedures for this problem, and showed that the ARE, under radial density $f$, of the normal-score version of their tests, with respect to the classical parametric Gaussian procedure (i.e., the standard multivariate portmanteau test), is given by

$$
\operatorname{ARE}_{k, f}^{(\mathrm{ser})}=\frac{1}{k^{4}}\left[D_{k}(\phi, f)\right]^{2}\left[C_{k}(\phi, f)\right]^{2}
$$

where

$$
D_{k}(\phi, f):=\mathrm{E}\left[\tilde{\Phi}_{k}^{-1}(U) \tilde{F}_{k}^{-1}(U)\right]
$$

see Hallin and Paindaveine [30].

\section{An elementary proof of multivariate Chernoff-Savage results.}

In this section, we give a unified and elementary proof that both families of ARE's in (2) and (4) above are uniformly - in $f$-larger than 1, which establishes the Pitman-inadmissibility of the corresponding standard Gaussian procedures (namely, Hotelling's test and the multivariate portmanteau test, respectively). We start with the inadmissibility result in the serial context :

Theorem 1 For all radial density $f$ such that $\mu_{k+1 ; f}<\infty$ and all positive integer $k$, we have $\operatorname{ARE}_{k, f}^{(\text {ser })} \geq 1$, where equality holds iff $f$ is Gaussian.

Let us now give the elementary proof we propose for this result, which allows for avoiding variational arguments, such as those used in Hallin and Paindaveine [30]. The proof, which is based on the method developed in Gastwirth and Wolff [2], does only make use of Jensen's inequality and-in the multivariate case - of the arithmetic-harmonic mean inequality.

Proof of Theorem 1. First rewrite the functional $f \mapsto C_{k}(\phi, f)$ defined in $(3)$ as 


$$
\begin{aligned}
C_{k}(\phi, f) & =\int_{0}^{\infty} \tilde{\Phi}_{k}^{-1}\left(\tilde{F}_{k}(r)\right) \varphi_{f}(r) \tilde{f}_{k}(r) d r \\
& =\frac{1}{\mu_{k-1 ; f}} \int_{0}^{\infty} \tilde{\Phi}_{k}^{-1}\left(\tilde{F}_{k}(r)\right)\left(-f^{\prime}(r)\right) r^{k-1} d r \\
& =\int_{0}^{\infty}\left[\frac{\tilde{f}_{k}(r)}{\tilde{\phi}_{k}\left(\tilde{\Phi}_{k}^{-1}\left(\tilde{F}_{k}(r)\right)\right)}+(k-1) \frac{\tilde{\Phi}_{k}^{-1}\left(\tilde{F}_{k}(r)\right)}{r}\right] \tilde{f}_{k}(r) d r
\end{aligned}
$$

where the last equality follows from integrating by parts. Applying first Jensen's inequality (with respect to the measure $\tilde{f}_{k}(r) d r$ and with convex function $g(x)=1 / x)$, and then the arithmetic-harmonic mean inequality, we obtain

$$
\begin{aligned}
C_{k}(\phi, f) & \geq\left\{\int_{0}^{\infty}\left[\frac{\tilde{f}_{k}(r)}{\tilde{\phi}_{k}\left(\tilde{\Phi}_{k}^{-1}\left(\tilde{F}_{k}(r)\right)\right)}+(k-1) \frac{\tilde{\Phi}_{k}^{-1}\left(\tilde{F}_{k}(r)\right)}{r}\right]^{-1} \tilde{f}_{k}(r) d r\right\}^{-1} \\
& \geq k^{2}\left\{\int_{0}^{\infty}\left[\frac{\tilde{\phi}_{k}\left(\tilde{\Phi}_{k}^{-1}\left(\tilde{F}_{k}(r)\right)\right)}{\tilde{f}_{k}(r)}+(k-1) \frac{r}{\tilde{\Phi}_{k}^{-1}\left(\tilde{F}_{k}(r)\right)}\right] \tilde{f}_{k}(r) d r\right\}^{-1} .
\end{aligned}
$$

Integrating by parts again yields

$$
\begin{aligned}
\int_{0}^{\infty} \tilde{\phi}_{k}\left(\tilde{\Phi}_{k}^{-1}\left(\tilde{F}_{k}(r)\right)\right) d r & =-\int_{0}^{\infty} r \frac{\tilde{\phi}_{k}^{\prime}\left(\tilde{\Phi}_{k}^{-1}\left(\tilde{F}_{k}(r)\right)\right)}{\tilde{\phi}_{k}\left(\tilde{\Phi}_{k}^{-1}\left(\tilde{F}_{k}(r)\right)\right)} \tilde{f}_{k}(r) d r \\
& =\int_{0}^{\infty} r\left[\tilde{\Phi}_{k}^{-1}\left(\tilde{F}_{k}(r)\right)-\frac{k-1}{\tilde{\Phi}_{k}^{-1}\left(\tilde{F}_{k}(r)\right)}\right] \tilde{f}_{k}(r) d r
\end{aligned}
$$

Substituting in (5), we obtain

$$
C_{k}(\phi, f) \geq k^{2}\left\{\int_{0}^{\infty} r \tilde{\Phi}_{k}^{-1}\left(\tilde{F}_{k}(r)\right) \tilde{f}_{k}(r) d r\right\}^{-1}=k^{2}\left[D_{k}(\phi, f)\right]^{-1}
$$

which establishes the inequality in Theorem 1.

Now, for the equality to hold, both Jensen's and arithmetic-harmonic mean inequalities need to be degenerate, i.e., we need to have

$$
\frac{\tilde{f}_{k}(r)}{\tilde{\phi}_{k}\left(\tilde{\Phi}_{k}^{-1}\left(\tilde{F}_{k}(r)\right)\right)}+(k-1) \frac{\tilde{\Phi}_{k}^{-1}\left(\tilde{F}_{k}(r)\right)}{r}=c, \forall r>0,
$$

and

$$
\frac{\tilde{\phi}_{k}\left(\tilde{\Phi}_{k}^{-1}\left(\tilde{F}_{k}(r)\right)\right)}{\tilde{f}_{k}(r)}=\frac{r}{\tilde{\Phi}_{k}^{-1}\left(\tilde{F}_{k}(r)\right)}, \forall r>0 .
$$


Equation (6) then can be rewritten as

$$
r^{1-k}\left[r^{k-1} \tilde{\Phi}_{k}^{-1}\left(\tilde{F}_{k}(r)\right)\right]^{\prime}=c, \forall r>0
$$

and holds iff $r^{k-1} \tilde{\Phi}_{k}^{-1}\left(\tilde{F}_{k}(r)\right)=a r^{k}+b$, for all $r>0$, for some real numbers $a, b$. Since the limit of $r^{k-1} \tilde{\Phi}_{k}^{-1}\left(\tilde{F}_{k}(r)\right)$ as $r$ goes to 0 is 0 , we must have $b=0$. This implies that $\tilde{\Phi}_{k}^{-1}\left(\tilde{F}_{k}(r)\right)=a r$, for all $r>0$, that is, $\tilde{\Phi}_{k}^{-1}(u)=a \tilde{F}_{k}^{-1}(u)$ for all $u \in(0,1)$, which means that $f$ is Gaussian (with arbitrary scale).

Finally, (7) is successively equivalent to

$$
\begin{gathered}
\tilde{f}_{k}\left(\tilde{F}_{k}^{-1}(u)\right) \tilde{F}_{k}^{-1}(u)=\tilde{\phi}_{k}\left(\tilde{\Phi}_{k}^{-1}(u)\right) \tilde{\Phi}_{k}^{-1}(u), \forall u \in(0,1), \\
\Leftrightarrow\left[\tilde{F}_{k}^{-1}(u)\right]^{\prime} / \tilde{F}_{k}^{-1}(u)=\left[\tilde{\Phi}_{k}^{-1}(u)\right]^{\prime} / \tilde{\Phi}_{k}^{-1}(u), \forall u \in(0,1), \\
\Leftrightarrow \tilde{F}_{k}^{-1}(u)=a \tilde{\Phi}_{k}^{-1}(u), \forall u \in(0,1),
\end{gathered}
$$

so that (7) holds iff $f$ is Gaussian (still with arbitrary scale).

The Pitman-inadmissibility of Hotelling's $T^{2}$ test for the multivariate onesample location problem now follows as a simple corollary. More precisely, we have the following :

Theorem 2 For all radial density $f$ such that $\mu_{k+1 ; f}<\infty$ and all positive integer $k$, we have $\operatorname{ARE}_{k, f}^{(\text {loc })} \geq 1$, where equality holds iff $f$ is Gaussian.

Proof of Theorem 2. The Cauchy-Schwarz inequality yields

$$
\left[D_{k}(\phi, f)\right]^{2} \leq D_{k}(\phi) D_{k}(f)=k D_{k}(f)
$$

so that $\mathrm{ARE}_{k, f}^{(\mathrm{loc})} \geq \mathrm{ARE}_{k, f}^{(\mathrm{ser})}$ for all radial density $f$. Consequently, the result follows from Theorem 1 . Note that equality holds in (8) iff $\tilde{F}_{k}^{-1}(u)=a \tilde{\Phi}_{k}^{-1}(u)$ $\forall u \in(0,1)$ for some $a>0$, that is, iff $f$ is Gaussian (with arbitrary scale).

In order to illustrate these Chernoff-Savage results, we consider two families of multivariate distributions, namely the class of $t$-distributions and the class of power-exponential distributions. Recall that the $k$-variate $t$-distribution with $\nu>0$ degrees of freedom is obtained by choosing, in (1), the radial density $f_{\nu}(r):=\left(1+r^{2} / \nu\right)^{-(k+\nu) / 2}, r>0$; the resulting elliptical distributions have heavier tails than the $k$-variate Gaussian distributions (note that the latter are obtained by letting $\nu$ go to infinity). In Figure 1, we present several plots, corresponding to various values of the space dimension $k$, of the ARE's in (2) and (4) under a broad class of $t$-distributions. These plots clearly provide an empirical verification (under heavy tails) of the Chernoff-Savage results in 
Theorems 1 and 2. Note that the ARE values in Figure 1 increase with the space dimension $k$. However, this is not a general phenomenon, as we show below by considering the class of power-exponential elliptical distributions.

The power-exponential elliptical distributions, which correspond to radial densities of the form $g_{\nu}(r):=\exp \left(-r^{2 \nu}\right)$, allow for considering tail weights (indexed by $\nu>0)$ that are heavier $(0<\nu<1)$ or lighter $(\nu>1)$ than in the normal case (which is obtained for $\nu=1$ ). In Figure 2, we give several plots of the ARE's in (2) and (4) under such power-exponential distributions, for various values of the space dimension $k$. Note that Figure 2 nicely illustrates the Chernoff-Savage results in Theorems 1 and 2 under heavy tails as well as under light tails, and that the ARE values are now decreasing with the space dimension $k$ (also note that the serial and location ARE curves in this case can extremely hardly be distinguished from each other).
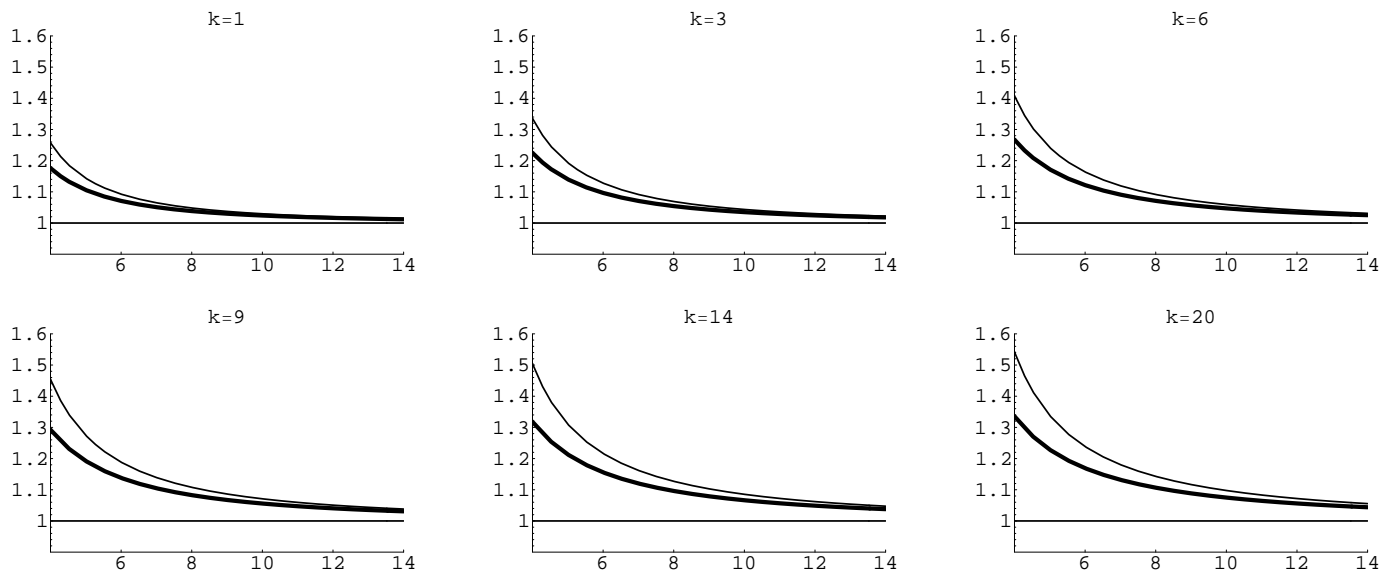

Fig. 1. Plots of $\mathrm{ARE}_{k, f_{\nu}}^{(\mathrm{loc})}$ (thin curve) and $\mathrm{ARE}_{k, f_{\nu}}^{(\mathrm{ser})}$ (thick curve) under $t$-distributions with $\nu$ degrees of freedom $(4<\nu<14)$, for various values of the space dimension $(k=1,3,6,9,14,20)$. The horizontal line corresponds to the performance of the Gaussian parametric procedure.
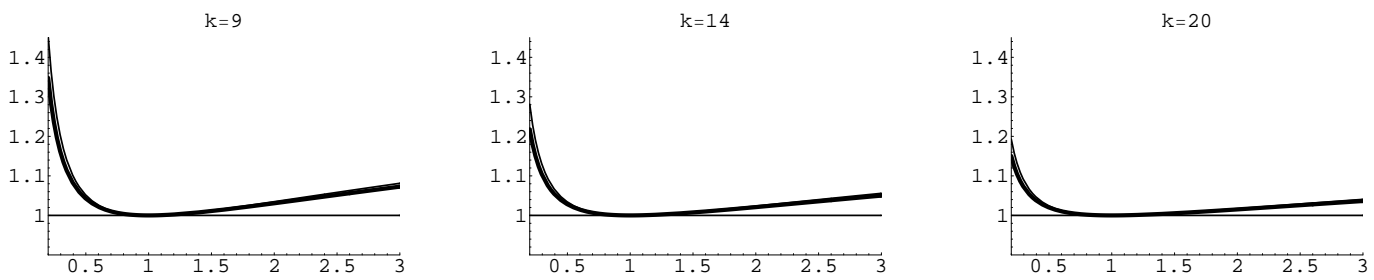

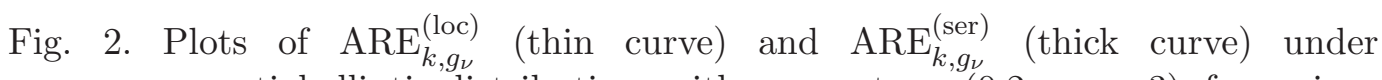
power-exponential elliptic distributions with parameter $\nu(0.2<\nu<3)$, for various values of the space dimension $(k=9,14,20)$. The horizontal line corresponds to the performance of the Gaussian parametric procedure. 


\section{Final comments.}

We would like to stress that the inadmissibility results in Theorems 1 and 2 are not confined to the problem of testing for multivariate randomness and to the multivariate one-sample location problem, respectively. Hallin and Paindaveine $[31,33]$ indeed extended their generalized signed-rank tests to more complicated models culminating in the multivariate general linear model with vector ARMA errors. The problems that can be dealt with are either associated with simple null hypotheses or with null hypotheses that are linear restrictions on the parameter of that very general model. Hallin and Paindaveine [31] showed that, in that context, the ARE's of the normal-score version of their tests, with respect to normal-theory competitors, are convex linear combinations of the ARE's in (2) and (4), obtained in the multivariate one-sample location case and in the problem of testing for multivariate randomness, respectively. Consequently, the Pitman-inadmissibility of parametric Gaussian procedures, as well as the simple proof provided above, extend to that very broad class of problems, which contains many problems of high practical relevance, such as multivariate Durbin Watson problems, ANOVA problems, the problem of testing the orders of a vector ARMA series, etc.

The methodology adopted in this paper can also be used to derive elementary proof of original Chernoff-Savage results. As an illustration, Paindaveine [34] considers the problem of testing for multivariate independence between two (elliptically symmetric) random vectors, and shows that the normal-score version of the rank-based tests recently proposed by Taskinen et al. [35] uniformly dominates - in the Pitman sense - the classical Wilks [36] test; the Pitmaninadmissibility of Wilks' test (hence, in the univariate case, of the classical correlation test) follows. To the best of our knowledge, even in the univariate case, whether the classical correlation test was admissible or not was so far an open question.

Also, for the sake of clarity, we have focused on hypothesis testing. But rankbased methods allow for dealing with point estimation as well. And it can be shown that the ARE's of the so-called R-estimators, with respect to the standard Gaussian estimators, do coincide with the ARE's obtained in the corresponding testing problems. So, e.g., in the multivariate one-sample case, the ARE's of the normal-score rank-based estimator of the location center, with respect to the multivariate average $\overline{\mathbf{X}}$ (the normal-theory competitor), are given in (2). Consequently, the generalized Chernoff-Savage results extend to this estimation problem, and the Pitman-inadmissibility of $\overline{\mathbf{X}}$ follows. More generally, this allows for establishing inadmissibility of multivariate least-squares and Yule-Walker estimators in the multivariate general linear model and in vector autoregressive models, respectively. 


\section{References}

[1] H. Chernoff, I. R. Savage, Asymptotic normality and efficiency of certain nonparametric tests, Ann. Math. Stat. 29 (1958) 972-994.

[2] J. L. Gastwirth, S. S. Wolff, An elementary method for obtaining lower bounds on the asymptotic power of rank tests, Ann. Math. Stat. 39 (1968) 2128-2130.

[3] J. L. Hodges, E. L. Lehmann, The efficiency of some nonparametric competitors of the $t$-test, Ann. Math. Stat. 27 (1956) 324-335.

[4] J. Pratt, J. Gibbons, Concepts of Nonparametric Theory, Springer Verlag, New York, 1981.

[5] I. Hájek, Z. Šidák, Theory of Rank Tests, Academic Press, New York, 1967.

[6] I. Hájek, Z. Šidák, P. Sen, Theory of Rank Tests, 2nd edition, Academic Press, New York, 1999.

[7] E. Lehmann, Testing Statistical Hypotheses, 2nd edition, John Wiley, New York, 1986.

[8] R. Randles, D. Wolfe, Introduction to the Theory of Nonparametric Statistics, John Wiley, New York, 1979.

[9] T. Hettmansperger, Statistical Inference Based on Ranks, John Wiley, New York, 1984.

[10] M. Puri, P. Sen, Nonparametric Methods in General Linear Models, John Wiley, New York, 1985.

[11] M. Hallin, J.-F. Ingenbleek, M. L. Puri, Linear serial rank tests for randomness against ARMA alternatives, Ann. Stat. 13 (1985) 1156-1181.

[12] M. Hallin, M. Puri, Optimal rank-based procedures for time-series analysis : testing an ARMA model against other ARMA models, Ann. Stat. 16 (1988) 402-432.

[13] M. Hallin, M. Puri, Time-series analysis via rank-order theory : signed-rank tests for ARMA models, J. Multivariate Anal. 39 (1991) 1-29.

[14] M. Hallin, M. Puri, Aligned rank tests for linear models with autocorrelated error terms, J. Multivariate Anal. 50 (1994) 175-237.

[15] M. Hallin, M. Puri, Rank tests for time-series analysis : a survey, In D. Brillinger, E. Parzen, and M. Rosenblatt Eds, New Directions in Time Series Analysis, Springer-Verlag, New York, 1992.

[16] M. Hallin, On the Pitman-nonadmissibility of correlogram-based methods, J. Times Series Anal. 15 (1994) 607-612.

[17] M. Puri, P. Sen, Nonparametric Methods in Multivariate Analysis, John Wiley, New York, 1971. 
[18] J. Möttönen, H. Oja, Multivariate spatial sign and rank methods, J. Nonparam. Statist. 5 (1995) 201-213.

[19] J. Möttönen, H. Oja, J. Tienari, On the efficiency of multivariate spatial sign and rank methods, Ann. Statist. 25 (1997) 542-552.

[20] J. Möttönen, T. P. Hettmansperger, H. Oja, J. Tienari, On the efficiency of the multivariate affine invariant rank methods, J. Multivariate Anal. 66 (1998) $118-132$.

[21] T. P. Hettmansperger, J. Nyblom, H. Oja, Affine invariant multivariate onesample sign tests, J. Roy. Statist. Soc. Ser. B 56 (1994) 221-234.

[22] T. P. Hettmansperger, J. Möttönen, H. Oja, Affine invariant multivariate onesample signed-rank tests, J. Amer. Statist. Assoc. 92 (1997) 1591-1600.

[23] H. Oja, Affine invariant multivariate sign and rank tests and corresponding estimates : a review, Scand. J. Statist. 26 (1999) 319-343.

[24] R. H. Randles, A distribution-free multivariate sign test based on interdirections, J. Amer. Statist. Assoc. 84 (1989) 1045-1050.

[25] R. H. Randles, A simpler, affine-invariant, multivariate, distribution-free sign test, J. Amer. Statist. Assoc. 95 (2000) 1263-1268.

[26] D. Peters, R. H. Randles, A multivariate signed-rank test for the one-sample location problem, J. Amer. Statist. Assoc. 85 (1990) 552-557.

[27] S.-L. Jan, R. H. Randles, A multivariate signed-sum test for the one-sample location problem, J. Nonparam. Statist. 4 49-63.

[28] R. H. Randles, Y. Um, Nonparametric tests for the multivariate multi-sample location problem, Statistica Sinica 8 (1998) 801-812.

[29] M. Hallin, D. Paindaveine, Optimal tests for multivariate location based on interdirections and pseudo-Mahalanobis ranks, Ann. Stat. 30 (2002) 1103-1133.

[30] M. Hallin, D. Paindaveine, Optimal procedures based on interdirections and pseudo-Mahalanobis ranks for testing multivariate elliptic white noise against ARMA dependence, Bernoulli 8 (2002) 787-815.

[31] M. Hallin, D. Paindaveine, Affine invariant aligned rank tests for the multivariate general linear model with ARMA errors, J. Multivariate Anal., to appear.

[32] H. Hotelling, The generalization of Student's ratio, Ann. Math. Stat. 2 (1931) $360-378$.

[33] M. Hallin, D. Paindaveine, Rank-based optimal tests of the adequacy of an elliptic VARMA model, Ann.stat., to appear.

[34] D. Paindaveine, Chernoff-Savage and Hodges-Lehmann results for Wilks' test of multivariate independence, submitted. 
[35] S. Taskinen, A. Kankainen, H. Oja, Rank scores tests of multivariate independence, University of Jyvaskyla, Preprint.

[36] S. S. Wilks, On the independence of $k$ sets of normally distributed statistical variables, Econometrica 3 (1935) 309-326. 